\title{
Modeling of Coating Stress of Plasma-sprayed Thermal Barrier Coatings
}

\author{
M. Arai $^{1, a}$ \\ 12-6-1 Nagasaka, Yokosuka-shi, Kanagawa-ken 240-0196 JAPAN \\ ${ }^{a}$ marai@criepi.denken.or.jp
}

Keywords: Gas Turbine, Thermal Barrier Coatings, Coating Stress, Shear-lag Theory

\begin{abstract}
The surfaces of gas turbine components are coated with thermal barrier coatings (TBCs) using a plasma spraying technique. A lot of effort has been expended examining the TBC interfacial strength, however studies examining how residual stress is formed after the process and how the coating stress changes with temperature are limited. In this report, the residual stress prediction model is proposed based on the splat deposition process. A simplified model including the plasma sprayed process is developed based on shear-lag theory. The simplification is given in continuous particle deposition process. That is, continuous particle deposited coating is modeled as a single layer, which is called by "deposition layer". This deposition layer is assumed to impact directly onto the substrate. The binding layer is also introduced to express multiple cracks caused by quenching stress in splats and sliding deformation at splat boundary. It is shown that the numerical analysis has good agreement with the associated experiments.
\end{abstract}

\section{Introduction}

Ceramic thermal barrier coatings (TBCs), which play an important role in insulating components such as blades of gas turbines from high heat flux and/or high-temperature environments, are generally deposited using plasma-spraying technology. Residual stress in TBCs, which is generated during the spraying process and in service, is a considerable mechanical factor in the performance and lifetime of the coated components. The residual stress could lead to cracking within the ceramic coating, and to spallation of ceramic coating brought by interfacial crack propagation. Thus, it is necessary to fully understand the residual stress in TBCs, in order to assess precisely the lifetime up to the occurrence of TBC failure.

A plasma spraying process is characterized by the impingement of molten particles onto a substrate and rapid solidification of the particles spread along the substrate. Here, we focus on single splat particle movement in plasma spraying process. Spherical-shaped molten particles are compressed into a high-temperature plasma flow and spread onto the surface of substrate. Shrinkage of the flattened particle (splat) is constrained by the underlying substrate, and a tensile microstress (quenching stress) is generated in the splat during cooling up to the point at which it reaches the substrate temperature $\left(T_{p}\right)$. The quenching stress can bring about cracking normal to the substrate surface forming a lamella structure which is a typical microstructure in TBCs. Another stress source, thermal stress occurs during cooling from the substrate temperature to room temperature $\left(T_{r}\right)$ after finishing a complete particle flattening and solidification process. Due to differences in thermal expansion, thermal stress develops in the dual-layered structure. This continuous deposition process gives the residual stress value in TBCs, and consequently it needs to be considered in a model for predicting the residual stress.

In this study, we develop a comprehensive numerical model to estimate residual stress in TBCs generated during the plasma spraying process, based on the assumption of layer build-up structure modeled as the continuous splat deposition process. The model is developed upon a shear lag theory for taking into account sliding deformation at splat boundaries and stress relaxation caused by 
cracking in splat. A strain gage method is employed to measure the residual stress. We compare the results predicted by our numerical model with experimental results in order to verify the proposed model.

\section{Modeling of Thermal Spray Deposition Process for TBC.}

Layer Build-up Model. In this study, a continuous molten particle deposition process is idealized by impingement of a single layer (called the "deposition layer".) onto a substrate. This idealized model representation is shown in Fig. 1. Residual stress comprises both quenching stress, which is caused by contraction of the deposition layer on the underlying substrate, and thermal stress, which is generated by a thermal expansion mismatch between the deposition layer and the substrate.

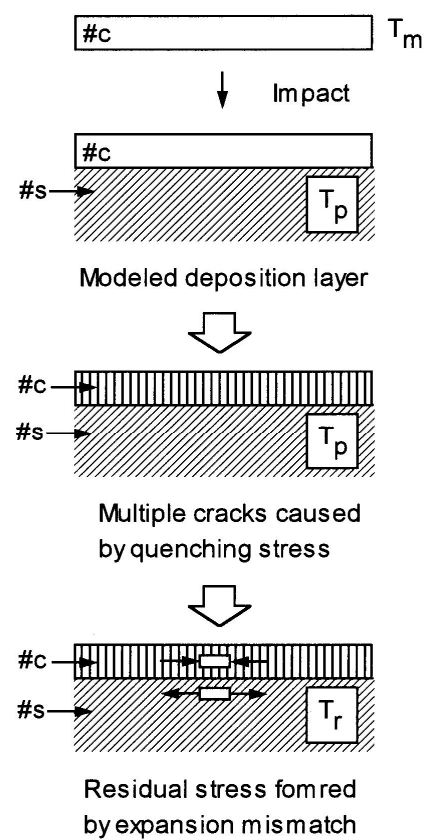

Fig.1 Schematic illustration for explanation of the model taking account of plasma -spraying process

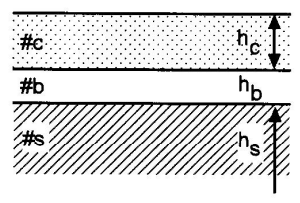

(a) Coated material

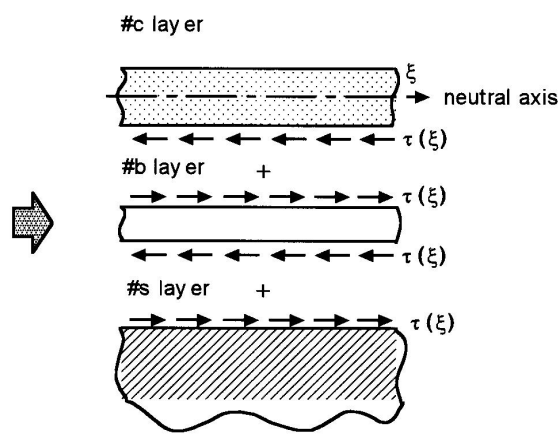

(b) Simplified mechanical model

Fig. 2 The model used for formulation of coating stress

The deposition layer formed into a high-temperature plasma flow with spray temperature $T_{m}$ impinges onto the substrate which is at a pre-heated temperature (substrate temperature) $T_{p}$ through the drifting force of the plasma gas flow. The deposition layer is in melting/liquid state if the temperature in the deposition layer is also the same as the spray temperature. This deposition layer deposits and spreads on the surface of the substrate when impacting onto the substrate. The temperature in the deposition layer is cooled quickly up to the point of reaching the substrate 
temperature, and a contraction of the deposition layer is generated on the underlying substrate. This contraction brings about quenching stress in the deposition layer. The quenching stress will be developed by a temperature drop from the solidification temperature $T_{s}$ to the substrate temperature $T_{p}$, because the effective temperature for causing thermal stress is limited in the region lower than the solidification temperature. The quenching stress generated during cooling will also cause cracking normal to the substrate surface as well as cracking phenomena observed in a single splat. After the temperature in the deposition layer reaches the substrate temperature, thermal stress arises in an overall dual-layered structure attached the deposition layer with a temperature drop from the substrate temperature to room temperature. Consequently, residual stress in TBC can be derived by summing the quenching stress and the thermal stress.

Formulation using Shear-lag Theory. The model utilized for formulation of coating stresses generated in the deposition layer during the plasma spraying process is shown in Fig. 2. Here, it should be noted that coating stress includes both quenching stress and thermal stress. In this study, a shear-lag theory is applied to obtain the coating stress, taking into account the fact that stress relaxation occurred in the deposition layer due to cracking.

Consequently, the coating stress generated in the deposition layer can be given by [1]:

$\sigma_{c}=\frac{N_{c}}{h_{c}}=\frac{\sinh \beta(\xi-l)-\sinh \beta \xi+\sinh \beta l}{\sinh \beta l} \frac{E_{c} E_{s} h_{s}}{E_{c} h_{c}+E_{s} h_{s}} \int_{T_{k}}^{T}\left(\alpha_{s}-\alpha_{c}\right) d T$

\section{Numerical Analysis Procedure.}

This section gives an explanation of the numerical analysis procedure to predict residual stress based on a layer build-up model.

Quenching stress analysis procedure:

(Step 1) Powder materials, solidification temperature and substrate temperature are set prior to the numerical analysis.

(Step 2) Deposition layer length is set to $l$ and coating stress is set to zero. Initial temperature in the deposition layer is set to solidification temperature $T_{s}$.

(Step 3) Incremental coating stress $\Delta \sigma_{c}$ for associated temperature change $\Delta T$ is calculated using the following equation:

$$
\Delta \sigma_{c}=\frac{\sinh \beta\left(\xi-\frac{l}{2^{i-1}}\right)-\sinh \beta \xi+\sinh \beta \frac{l}{2^{i-1}}}{\sinh \beta \frac{l}{2^{i-1}}} \frac{E_{c} E_{s} h_{s}}{E_{c} h_{c}+E_{s} h_{s}}\left(-\alpha_{c}\left(T_{i}\right)\right) \Delta T
$$

where $i$ indicates the number of incremental calculations $(i=1,2, \ldots \ldots)$ and $T_{i}=T_{i-1}+\Delta T$. The term $\left(\frac{l}{2^{i-1}}\right)$ means length divided by cracking generated in the deposition layer.

(Step 4) The coating stress at step $i$ can be obtained from $\sigma_{c}^{i}=\sigma_{c}^{i-1}+\Delta \sigma_{c}$. If this coating stress does not reach the fracture strength $\sigma_{f}$ of the ceramic material or the temperature $T_{i}$ is larger than the substrate temperature $T_{p}$, the incremental calculation again returns to step (3).

(Step 5) If the coating stress exceeds the fracture strength, step $n$ is progressed to $n+1$ by cracking initiation in the deposition layer. The coating stress at step $i=n+1$ is calculated using:

$$
\sigma_{c}^{i}=\frac{\sinh \beta\left(\xi-\frac{l}{2^{n}}\right)-\sinh \beta \xi+\sinh \beta \frac{l}{2^{n}}}{\sinh \beta \frac{l}{2^{n}}} \frac{E_{c} E_{s} h_{s}}{E_{c} h_{c}+E_{s} h_{s}} \int_{T_{k}}^{T_{i}}\left(-\alpha_{c}\right) d T
$$


and then the subsequent calculation returns to step (3) with Eq. (3).

Thermal stress analysis procedure:

(Step 6) The incremental coating stress $\Delta \sigma_{c}$ for the associated incremental temperature $\Delta T$ can be obtained by,

$$
\Delta \sigma_{c}=\frac{\sinh \beta\left(\xi-\frac{l}{2^{N}}\right)-\sinh \beta \xi+\sinh \beta \frac{l}{2^{N}}}{\sinh \beta \frac{l}{2^{N}}} \frac{E_{c} E_{s} h_{s}}{E_{c} h_{c}+E_{s} h_{s}}\left(\alpha_{c}\left(T_{i}\right)-\alpha_{c}\left(T_{i}\right)\right) \Delta T
$$

where $N-1$ is final number of incremental calculations after the quenching stress analysis.

(Step 7) The coating stress at step $i$ is obtained from $\sigma_{c}^{i}=\sigma_{c}^{i-1}+\Delta \sigma_{c}$. If the temperature $T_{i}$ is higher than room temperature $T_{r}$, the calculation returns to step (6). If the temperature $T_{i}$ reaches room temperature, all calculations are finished. The coating stress at the final step consequently gives the residual stress in TBC.

\section{Material Constants for Numerical Analysis.}

Material constants are summarized as following;

(1) Substrate (Type 304 stainless steel)

$$
\begin{array}{ll}
\alpha_{s}(T) & =14.644+9.1534 \times 10^{-3} T-5.149 \times 10^{-6} T^{2}+5.6839 \times 10^{-10} T^{3} \quad\left[\times 10^{-6} 1 / K\right] \\
E_{s}(T) & =200 \quad[G P a]
\end{array}
$$

(2) Deposition layer (ceramic coating)

$$
\begin{aligned}
\alpha_{c}(T) & =3.8256+1.6493 \times 10^{-3} T-1.2514 \times 10^{-5} T^{2}+3.1118 \times 10^{-3} T^{3} \quad\left[10^{-6} 1 / K\right], \quad T<1000[K] \\
& =10.916 \quad\left[10^{-6} 1 / K\right], \quad T \geq 1000[K] \\
E_{c}= & 200 \quad[\mathrm{GPa}] \\
\sigma_{f}= & 6159 \times \exp (-0.0039503 T) \quad[\mathrm{MPa}]
\end{aligned}
$$

(3) Binding layer

$$
\begin{aligned}
& h_{b}=h_{c} / 5 \\
& \mu_{b}\left(v_{p}\right)=0.0078127 v_{p}^{2}
\end{aligned}
$$

Other analysis parameters include, initial length of deposition layer of $25[\mathrm{~mm}]$, the thickness of layer $h_{c}$ equals $1[\mathrm{~mm}]$ and the substrate thickness $h_{s}$ is $5[\mathrm{~mm}]$. Poisson's ratio $v_{c}$ for deposition layer takes 0.07 , which is same as one of freestanding ceramic coating, and one of substrate $v_{s}$ is 0.3 . In this case, solidification temperature $T_{s}$ was assumed to be $2000[\mathrm{~K}]$. This assumption is also reasonable from particle temperature measurement and associated material handbook data [2].

\section{Numerical Analysis Results}

Typical result for variation of coating stress with temperature is indicated in Fig.3. These result corresponds to the following plasma spraying condition; $T_{p}=600[\mathrm{~K}]$ and $v_{p}=175[\mathrm{~m} / \mathrm{s}]$. The broken line in this graph indicates the fracture strength curve of the sintering ceramic material [3]. It was found that the coating stress in deposition layer increased with temperature reducing from solidification temperature. The coating stress reaches the fracture strength and then causes crack in 
this layer. This coating stress or quenching stress in the plasma-spraying process is as described previously. The cracking reduces the coating stress in the deposition layer. The coating stress increases again with reduction of temperature, and again reaches the fracture strength of the sintering ceramic, which causes cracking in the deposition layer. Increase of coating stress, initiation of crack and drop of coating stress cyclically rise up to reaching to approximately $1700[\mathrm{~K}]$. Multiple cracks, which were caused by the coating stress, lead to decrease of elastic modulus of deposition layer. Thus, the coating stress varies with lower slope against decreasing temperature. After reaching the substrate temperature, the coating stress in the deposition layer shifts to compressive due to thermal expansion mismatch between deposition layer and substrate. Finally, we have residual stress formed in the deposition layer, that is, in TBC. This process below substrate temperature corresponds to thermal stress process. This figure provides that the proposed simplified model based on plasma-spraying process gives us nice result for coating stress simulation in TBC.

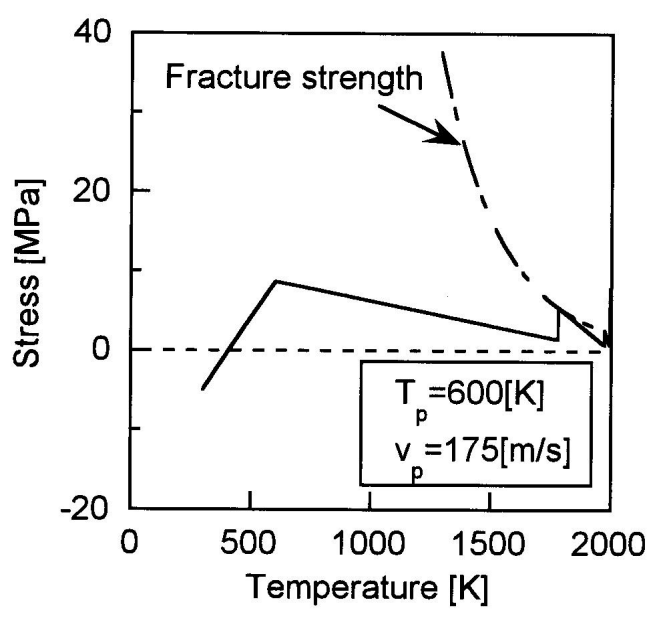

Fig. 3 Simulation result of coating stress changing with temperature in case of $T p=600[\mathrm{~K}]$ and $v_{p}=175[\mathrm{~m} / \mathrm{s}]$

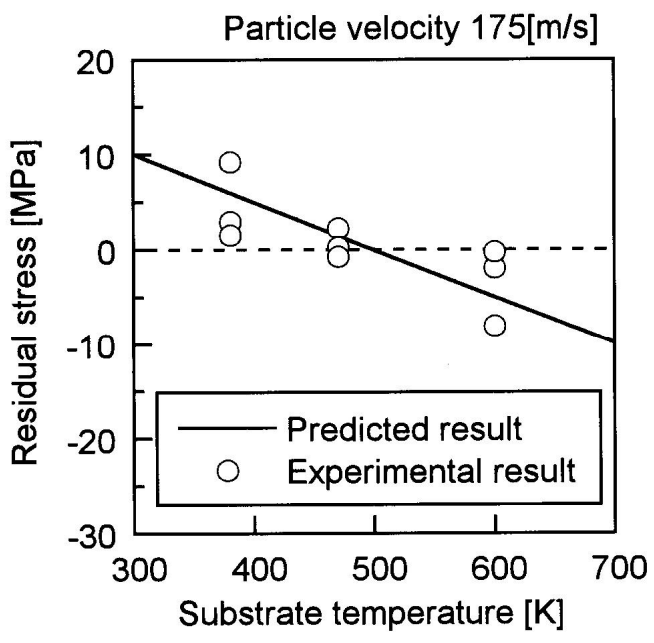

Fig.4 Comparison between numerical and experimental results in residual stress versus substrate temperature condition

Relationship between residual stress and substrate temperature condition is indicated in Fig.4. In this figure, the circles indicate experimental results measured by the strain gage method. The solid line represents the numerical result from the simplified model. It is found that this comparison between experiment and numerical results gives good agreement over all substrate temperature condition. Relationship between residual stress and particle velocity condition is also shown in Fig.5. We can 
say that the experimental results almost correspond to the numerical one in this case, however some differences at higher particle velocities were observed.

\section{Conclusion}

In this report, the simplified model was developed in consideration of the spraying deposition process. The obtained results are summarized as following; the deposition process was modeled to simulate residual stress in TBC. Modeling is divided into two following processes; quenching stress process caused by contraction of splat when molten particle impacting onto the substrate, and thermal stress by thermal expansion mismatch. Simplification was achieved for continuous particle deposition by introducing a deposition layer. Another simplification was achieved by introduce a binding layer between the deposition layer and substrate, which models sliding deformation at splat boundary and multiple cracking in the coating caused by quenching stress. Numerical analysis was conducted for as-sprayed TBC. The numerical results showed good agreement with experimental one. In this study, temperature distribution within the TBC was not taken into which would have an effect. Therefore we need to improve the model taking into account the effect of temperature distribution and associated stress distribution. However, we believe that the proposed model is quite simple and easy understood for engineers within the thermal spraying field, enabling them to choose the optimum particle energy and substrate temperature.

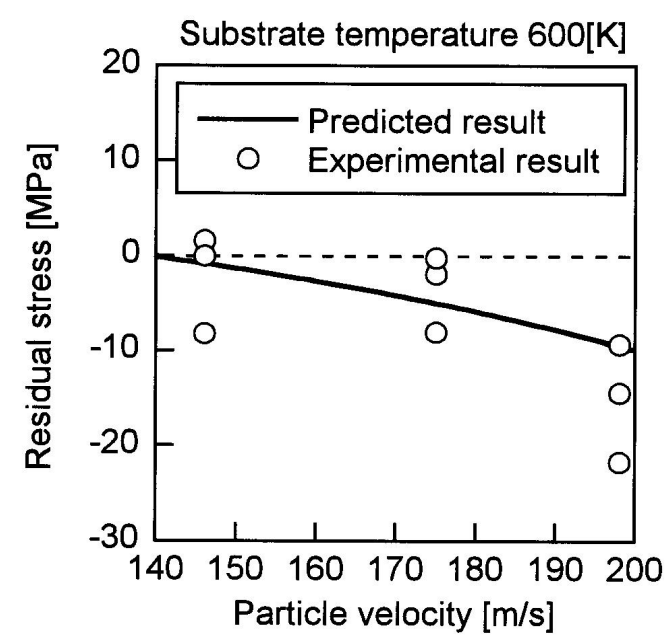

Fig.5 Comparison between numerical and experimental results in residual stress versus particle velocity condition

\section{Acknowledgements}

The author would like to acknowledgement for Mr. Ohno of Plasma Giken co. 1td. for conducting plasma-spraying process of $\mathrm{TBC}$, and also the helpful comments for particle deposition process mechanism given by Dr. Fukanuma of the same company. Those experiments were conducted by $\mathrm{Mr}$. Wada of Tokyo Institute of Technology. The author also would like to say thank you for him.

\section{References}

[1] M. Arai, E. Wada, K. Kishimoto: J. of Solid Mech. and Mat. Eng., Vol. 1, No. 10 (2007), pp.1251-1261.

[2] D. J. Green, R. H. J. Hnnink, M. V. Swain: "Transformation Toughening of Ceramics", CRC Press, Inc. (1989), for instance.

[3] R. Fujisawa, K. Matsusue, K. Kitahara: J. of the Society of Mat. Sci. Jpn, Vol. 35 (1986), pp.1112-1117. 